\title{
Backward-forward reaction asymmetry of neutron elastic scattering on deuterium
}

\author{
E. Pirovano, ${ }^{1}$ R. Beyer,${ }^{2}$ A. R. Junghans,${ }^{2}$ N. Nankov,${ }^{1}$ R. Nolte,${ }^{3}$ M. Nyman,${ }^{1}$ and A. J. M. Plompen ${ }^{1, *}$ \\ ${ }^{1}$ European Commission, Joint Research Centre, Retieseweg 111, 2440 Geel, Belgium \\ ${ }^{2}$ Helmholtz-Zentrum Dresden-Rossendorf, Institute of Radiation Physics, Bautzner Landstraße 400, 01328 Dresden, Germany \\ ${ }^{3}$ Physikalisch-Technische Bundesanstalt, Department 6.4 - Ion and Neutron Radiation, Bundesallee 100, 38116 Braunschweig, Germany
}

(Received 25 May 2016; revised manuscript received 28 September 2016; published 2 February 2017)

\begin{abstract}
A new measurement of the angular distribution of neutron elastic scattering on deuterium was carried out at the neutron time-of-flight facility nELBE. The backward-forward asymmetry of the reaction was investigated via the direct detection of neutrons scattered at the laboratory angle of $15^{\circ}$ and $165^{\circ}$ from a polyethylene sample enriched with deuterium. In order to extend the measurement to neutron energies below $1 \mathrm{MeV},{ }^{6} \mathrm{Li}$ glass scintillators were employed. The data were corrected for the background and the multiple scattering in the target, the events due to scattering on deuterium were separated from those due to carbon, and the ratio of the differential cross section at $15^{\circ}$ and $165^{\circ}$ was determined. The results, covering the energy range from $200 \mathrm{keV}$ to $2 \mathrm{MeV}$, were found to be in agreement with the theoretical predictions calculated by Canton et al. [Eur. Phys. J. A 14, 225 (2002)] and by Golak et al. [Eur. Phys. J. A 50, 177 (2014)]. The comparison with the evaluated nuclear data libraries indicated CENDL-3.1, JEFF-3.2, and JENDL-4.0 as the evaluations that best describe the asymmetry of $n$ - $d$ scattering. ENDF/B-VII.1 is compatible with the data for energies below $700 \mathrm{keV}$, but above the backward to forward ratio is higher than measured. ROSFOND-2010 and BROND-2.2 resulted to have little compatibility with the data.
\end{abstract}

DOI: 10.1103/PhysRevC.95.024601

\section{INTRODUCTION}

Neutron scattering on deuterium ${ }^{1}$ is one of the simplest cases of the many body problem in nuclear physics and, as such, it is a valuable means of investigation of the fundamental interactions between nucleons. It is, moreover, a case study of practical interest for nuclear applications. In nuclear engineering, for instance, the interest mainly concerns the operation of heavy-water moderated reactors. In neutron metrology, the $n-d$ scattering cross section is a necessary information as it determines the energy distribution of the $\mathrm{D}_{2} \mathrm{O}$-moderated ${ }^{252} \mathrm{Cf}$ fission neutron reference field, which is used for example for the calibration of neutron dosimeters [1]. In detector physics, the thorough knowledge of the reaction is crucial for the proper characterization of $\mathrm{C}_{6} \mathrm{D}_{6}$ scintillation detectors, whose response to neutrons depends indeed on $n$ - $d$ scattering [2].

The differential cross section of neutron elastic scattering on deuterium is an item in the High-Priority Request List of the OECD-NEA Data Bank for nuclear data measurements [3]. The request is motivated by the fact that the experimental angular distributions available in EXFOR [4] are scarce and partially inconsistent, particularly at angles near $180^{\circ}$, and the measurements are 25 to more than 50 years old [5]. Moreover, the energy-angle evaluated probability distributions have been found to cause inconsistencies when trying to reproduce the

*arjan.plompen@ec.europa.eu

Published by the American Physical Society under the terms of the Creative Commons Attribution 3.0 License. Further distribution of this work must maintain attribution to the author(s) and the published article's title, journal citation, and DOI.

${ }^{1}$ Throughout the text ' $\mathrm{D}$ ' denotes '2 $\mathrm{H}$ ' or deuterium. results of benchmark experiments for heavy-water moderated critical assemblies [6,7].

In 2006, Townsend [5] reviewed the experimental $n$ - $d$ cross section data in the energy range relevant for fission reactions and the ENDF/B-VI.8 [8], JENDL-3.3 [9], and JEFF-3.1 [10] evaluations. The total elastic cross sections in ENDF/B-VI.8 and JEFF-3.1 were found to be identical, with JENDL-3.3 differing from them by less than $1 \%$. However when angular distributions were considered, significant inconsistencies were noticed, particularly at backwards angles. In the range from $220 \mathrm{keV}$ to $3.2 \mathrm{MeV}$ the evaluations were compared to the experimental differential cross section, but an overall poor agreement was found.

In 2002 Canton et al. studied the consequences of introducing in the three-nucleon potential the irreducible effects generated by the one-pion-exchange mechanism [11]. They presented the theoretical predictions of the cross section and other observables of the nucleon-deuteron scattering in the energy range from 3 to $19 \mathrm{MeV}$. In 2007 Svenne et al. extended these calculations further down to $50 \mathrm{keV}$ [12], and used the resulting differential cross section to provide additional information to compare to existing datasets the ENDF/B-VII.0 [13] and JENDL-3.3 libraries. Their results were found to have a better agreement with JENDL-3.3, while the biggest difference with ENDF/B-VII.0 was found at backward angles.

More recently, the nucleon-deuteron scattering has been studied in the framework of effective field theory (EFT) [14-16]. In these works, the $n$ - $d$ scattering has been mainly used as means of validation of the three-nucleon potential derived via chiral perturbation theory applied to low-energy quantum chromodynamics. In Ref. [15], for instance, Golak et al. computed the three-nucleon forces at the fourth order (next-to-next-to-next-to the leading order, N3LO) of the expansion, and applied the full N3LO Hamiltonian to 
$n$ - $d$ elastic scattering and breakup reactions. For incoming nucleon energies below $20 \mathrm{MeV}$, discrepancies were observed between the EFT predictions of the spin observables and the experimental data. However, for the elastic scattering angular distribution in the same energy range, the effects of the three-nucleon forces were found to be negligible (see also [16]), and the theory agrees well with the data.

Since the $n-d$ scattering total cross section is already well known, the present work focused only on the study of the backward-forward asymmetry of the reaction. The results are here presented of an experiment where the cross section ratio between the laboratory angles of $165^{\circ}$ and $15^{\circ}$ was determined. The aim was to provide a comparison between the main nuclear data libraries, theoretical calculations and new experimental data, therefore the two angles $165^{\circ}$ and $15^{\circ}$ were chosen in order to maximize the difference between evaluations.

The following libraries were considered: BROND-2.2 [17], CENDL-3.1 [18], JEFF-3.2 [19], JENDL-4.0 [20], ENDF/BVII.1 [21], and ROSFOND-2010 [22]. All evaluations but ROSFOND-2010, which is based on ENDF/B-VI.3, result from independent analyses. CENDL-3.1, JEFF-3.2, and JENDL-4.0 are based on three-body models and the solution of the Faddeev equation; in ENDF/B-VII.1 the elastic angular distributions are the results of a coupled-channels $R$-matrix analysis.

In the experiment, performed at the nELBE neutron timeof-flight facility $[23,24]$, neutrons scattered from a deuterated polyethylene $\left(\mathrm{CD}_{2}\right)$ sample were directly detected using lithium-6 enriched glass scintillators. As energies of interest range from a few hundred $\mathrm{keV}$ to few $\mathrm{MeV}$, detectors such as organic scintillators, e.g., NE213 detectors, that use scattering on hydrogen as conversion reaction for neutron detection, were not considered suitable because of the strong quenching of the scintillation efficiency for neutron energies below $1 \mathrm{MeV}$ and the related decrease of the efficiency for a given detection threshold. However, because of the low detection efficiency, it was necessary to have more than one detector at the selected positions, so in practice it was not possible to consider more than two angles.

A similar experiment was already attempted once before [25], but the amount of background due to room return neutrons made the analysis difficult and the poor statistics resulted in large uncertainties. After the enlargement of the nELBE experimental hall, the measurement was repeated, and with the room background significantly decreased it was possible to cover the energy range from $200 \mathrm{keV}$ to $2 \mathrm{MeV}$.

\section{EXPERIMENTAL SETUP}

nELBE is the neutron time-of-flight facility installed at ELBE, the superconducting Electron Linac for beams with high Brilliance and low Emittance of the Helmholtz-Zentrum Dresden-Rossendorf [26,27]. At nELBE [28], neutrons are produced by an electron beam impacting on a liquid lead target, via $(\gamma, n)$ reactions induced by bremsstrahlung. Since the neutrons are emitted almost isotropically while the bremsstrahlung is forward peaked, the neutron beam is defined by a collimator: a steel tube with lead and polyethylene inserts, installed at $95^{\circ}$ with respect to the primary electron beam. Different absorbers

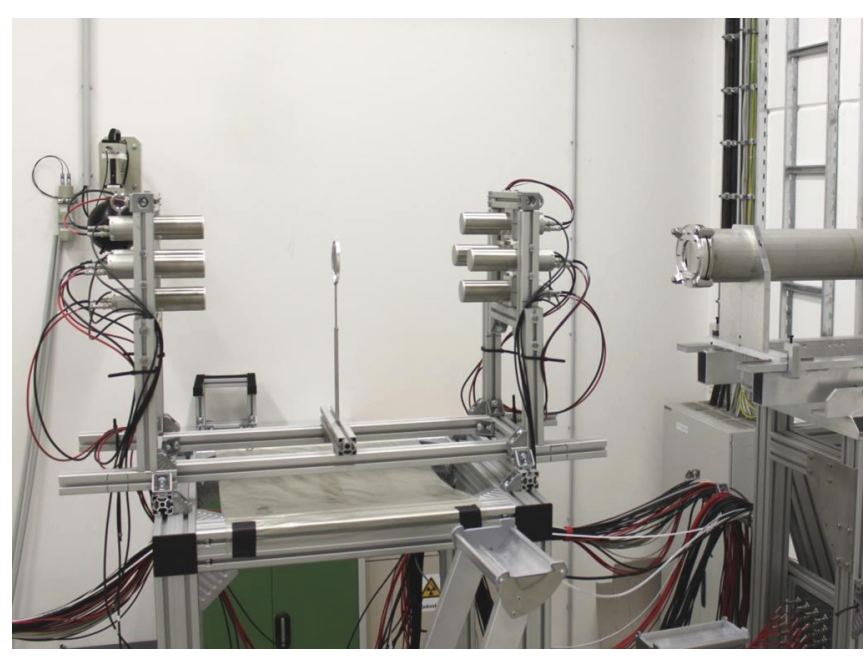

FIG. 1. Picture of the detector array.

can be set up in front of the collimator in order to adjust the beam properties, such as the $\gamma$-flash intensity. In this experiment, $3 \mathrm{~cm}$ of lead were used. The experimental hall is separated from the neutron radiator by a $2.5 \mathrm{~m}$ thick wall of heavy and normal concrete, and the detector setup is located at least at $3 \mathrm{~m}$ from the walls, the ceiling and the floor.

The setup used for the experiment is shown in Fig. 1 and schematized in Fig. 2. Eight cylindrical lithium glass detectors enriched in lithium-6 from Scionix (model 51 B 12,7/2M-O-E1-LiG-Neg-X, see Table I for the specifics) were mounted on an aluminium frame, in two groups of four, at $165^{\circ}$ and $15^{\circ}$ to the beam axis. The sample was positioned in the middle of the frame centered on the beam

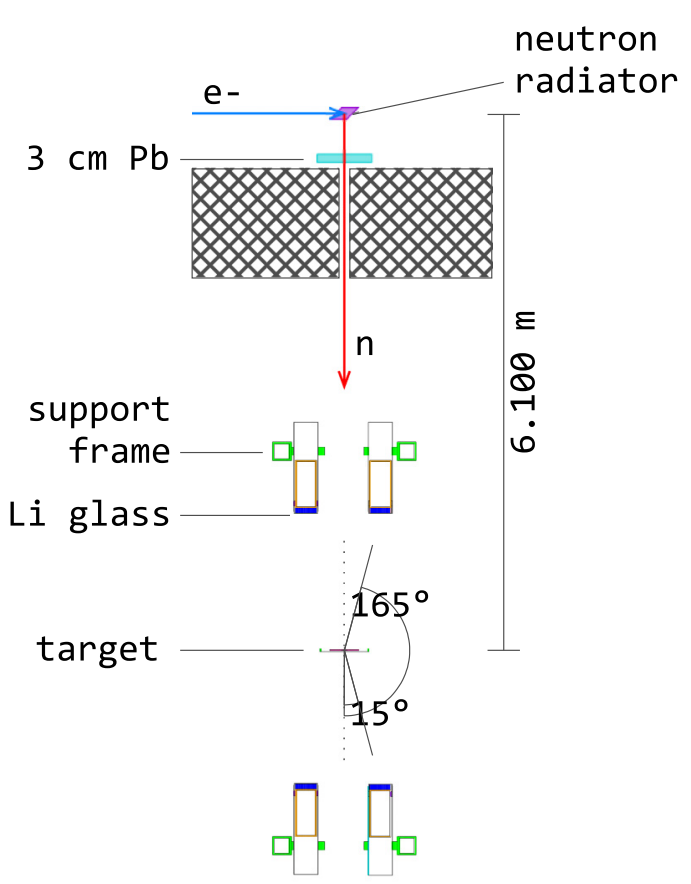

FIG. 2. Diagram of the experimental setup (horizontal plane passing through the center of the target). 
TABLE I. Lithium glass specifications by Scionix.

\begin{tabular}{lcc}
\hline \hline dimensions: & $\begin{array}{c}\text { diameter } \\
\text { thickness }\end{array}$ & $\begin{array}{c}50.8 \mathrm{~mm} \\
12.7 \mathrm{~mm}\end{array}$ \\
\hline composition: & $\mathrm{SiO}_{2}$ & $56 \%$ \\
(\% in weight) & $\mathrm{MgO}$ & $4 \%$ \\
& $\mathrm{Al}_{2} \mathrm{O}_{3}$ & $18 \%$ \\
& $\mathrm{Ce}_{2} \mathrm{O}_{3}$ & $4 \%$ \\
& ${ }^{6} \mathrm{Li}_{2} \mathrm{O}$ & $18 \%$ \\
lithium-6 content: & \multicolumn{2}{c}{$7.4 \%$ weight } \\
density: & \multicolumn{2}{c}{$2.5-2.7 \mathrm{~g} / \mathrm{cm}^{3}$} \\
\hline \hline
\end{tabular}

axis, 6.100(1) $\mathrm{m}$ from the neutron source. Two different samples were used as targets: a deuterated polyethylene disk with $99.999 \%$ enrichment in deuterium, and a graphite disk which was used to assess the contribution of carbon in the $\mathrm{CD}_{2}$ measurement. A measurement with the empty sample holder was also performed to determine the background due to neutrons scattering in air or in the supporting frame. The specifics of the samples and the position of the detectors are reported in Tables II and III, respectively. A graphite sample three times thicker than the $\mathrm{CD}_{2}$ target was chosen in order to avoid the problems encountered when carrying out the experiment the first time, when the measurements with a thinner carbon sample could not be used because of the amount of room return background.

The data acquisition (DAQ) system (diagram in Fig. 4) was based on commercially available VME modules read out by a CES RIO4 VME Power-PC running the real time operating system LynxOS and the DAQ software MBS developed by GSI Darmstadt [29]. The signal of each detector was split by a 50-Ohm-splitter and then fed to a charge-to-digital converter (QDC, type CAEN V965A) and a constant fraction discriminator (CFD, in house development of HZDR). The output of the CFD was fed to a scaler (realized by an FPGA module type CAEN V1495), a time-to-digital converter (TDC, type CAEN V1290A) and a trigger logic module (implemented in a second FPGA module type CAEN V1495). The latter generated the trigger for both the QDC and the TDC, and accomplished the dead time logic. The TDC gathered the signals from both the detectors and the accelerator reference signal, determining in this way the time-of-flight (t.o.f.). The dead time of the DAQ system, i.e., the time needed for the analog-to-digital conversion of the signals and the read-out of the buffer memories, was determined integrally by the scaler and per event by the trigger logic, using a VETO signal that was the logical OR of the busy signals of all electronic modules. Thereby a t.o.f. dependent dead time correction could be applied using the procedure described in Ref. [28].

TABLE II. Physical properties of the samples.

\begin{tabular}{llc}
\hline \hline & $\mathrm{CD}_{2}$ & graphite \\
\hline diameter $(\mathrm{cm})$ & $7.00(2)$ & $11.0(1)$ \\
thickness $(\mathrm{cm})$ & $0.30(2)$ & $0.93(2)$ \\
areal density $\left(\mathrm{g} / \mathrm{cm}^{2}\right)$ & $0.3236(9)$ & $1.52(1)$ \\
\hline \hline
\end{tabular}

TABLE III. Position of the detectors. The distances $x, y, z$, and $d$ and the angle $\theta$, defined as described in Fig. 3, refer to position of the center of the detector external housing's front face with respect to the beam axis or the center of the target. The angles covered by the whole front surface are in the range of $\pm 4^{\circ}$ the angle measured at its center.

\begin{tabular}{lccrr}
\hline \hline$x(\mathrm{~mm})$ & $|y|$ or $|z|(\mathrm{mm})$ & $d(\mathrm{~mm})$ & $\cos \theta$ & $\theta\left(^{\circ}\right)$ \\
\hline $322(2)$ & $86(1)$ & $333(2)$ & $0.9661(8)$ & $15.0(2)$ \\
$320(2)$ & $92(1)$ & $333(2)$ & $0.9611(9)$ & $16.0(2)$ \\
$324(2)$ & $87(1)$ & $336(2)$ & $0.9658(8)$ & $15.0(2)$ \\
$320(2)$ & $88(1)$ & $332(2)$ & $0.9642(9)$ & $15.4(2)$ \\
$-327(2)$ & $83(1)$ & $338(2)$ & $-0.9693(8)$ & $165.8(2)$ \\
$-327(2)$ & $95(1)$ & $340(2)$ & $-0.9603(9)$ & $163.8(2)$ \\
$-328(2)$ & $87(1)$ & $339(2)$ & $-0.9669(8)$ & $165.2(2)$ \\
$-328(2)$ & $92(1)$ & $341(2)$ & $-0.9629(9)$ & $164.3(2)$ \\
\hline \hline
\end{tabular}

The present experiment did not aim at measuring the full angular distribution but only the backward-forward asymmetry at a selected pair of forward and backward angles. For this reason and because the relative neutron energy distribution was already determined previously using a ${ }^{235} \mathrm{U}$ fission chamber [28], a precise flux determination was not required. To compare the runs with different targets and the "sample-out" run, the relative beam fluence was derived from the total counts of a plastic scintillator installed downstream with respect to the setup. The scintillator is mainly sensitive to scattered photons, therefore its counting rate depended on the sample in place at a given moment. The differences were quantified by assuming that the beam flux would remain constant during the time needed to change or remove the sample. This was deemed reasonable because the accelerator current, measured continuously upstream, was notably stable during the whole experiment, and the procedure to change target needed about half an hour to be completed. Therefore, the difference in the counting rate of the monitor at the end of one run and the

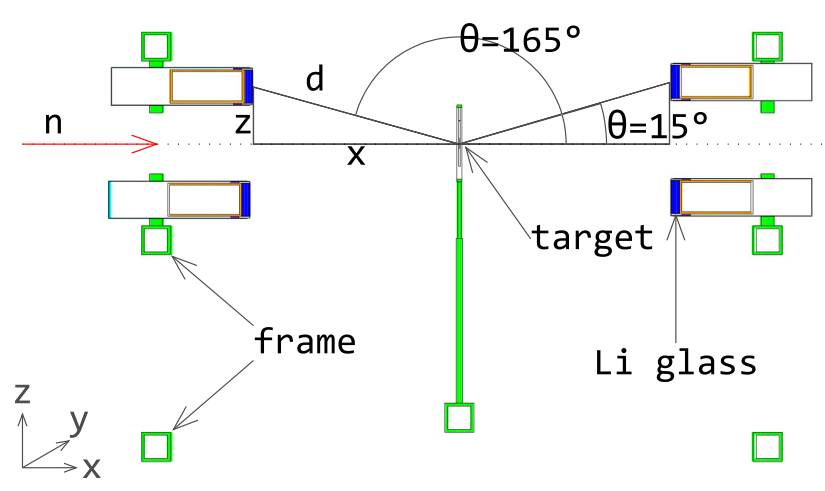

FIG. 3. Model of the detectors, the target, and the supporting aluminium frame implemented in MCNP5. Here the vertical $x-z$ plane passing through the center of the target is shown; an analogous figure is obtained when the $x-y$ plane is plotted, with the difference that there the distance $z$ is replaced by that along the $y$ axis. The distances $x, y$, and $z$ were actually measured, $d$ and the angle $\theta$ were computed accordingly. The exact values of $x, y$ or $z, d$, and $\theta$ are reported in Table III. 


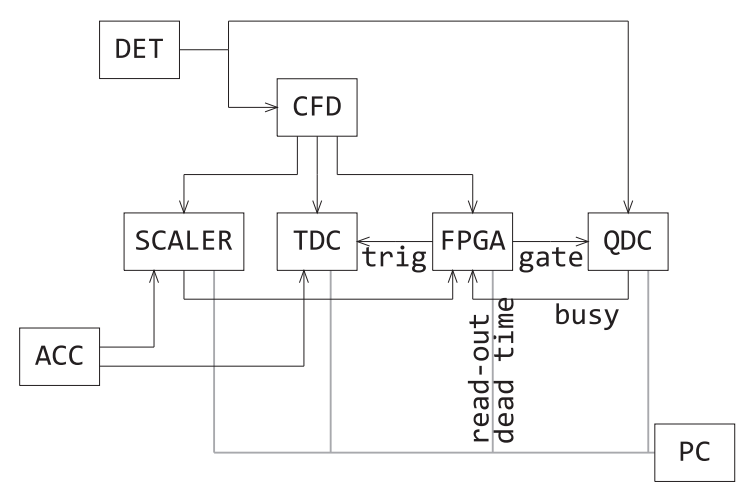

FIG. 4. Diagram of the data acquisition system. DET: Li-glass detector; ACC: accelerator reference signal; CFD: constant fraction discriminator; FPGA: field programmable gate array logic module; QDC: charge-to-digital converter; TDC: time-to-digital converter; SCALER: scaler module.

beginning of the following was caused only by the difference in the sample. The ratio between the two values was used to normalize the monitor counts of the graphite run and sample-out run to the $\mathrm{CD}_{2}$ run.

\section{ANALYSIS OF THE TIME-OF-FLIGHT SPECTRA}

In facilities such as nELBE, where an electron linac is used to produce neutrons, time-of-flight experiments are accomplished by operating the accelerator in pulsed mode and by measuring the elapsed time between the neutron production and their detection. In the case of ELBE, the electrons are produced in pulses of 5 ps width (FWHM) and kinetic energy up to $40 \mathrm{MeV}$; the repetition rate can reach up to $26 \mathrm{MHz}$, but for the production of neutrons it is typically set between 100 to $250 \mathrm{kHz}$ [28] (101 kHz in this experiment).

In this experiment, the measured time-of-flight consisted of the sum of the time-of-flight of the incoming neutron traveling from the source to the scattering target, and the time-of-flight of the scattered neutron going from the target to the detector. The relationship between time-of-flight (t.o.f.) and neutron energy before and after the collision $\left(E\right.$ and $\left.E^{\prime}\right)$ could then be expressed as

$$
\text { t.o.f. }=\frac{L}{c \sqrt{1-\frac{1}{\left(1+E / m c^{2}\right)^{2}}}}+\frac{L^{\prime}}{c \sqrt{1-\frac{1}{\left(1+E^{\prime} / m c^{2}\right)^{2}}}},
$$

where $c$ is the speed of light, $m$ the neutron mass, $L$ and $L^{\prime}$ the length of the flight paths, respectively, of the incident and scattered neutron. In case of elastic scattering on a given nuclide, the energy after the collision $E^{\prime}$ depends on the energy $E$ and on the scattering angle $\theta$. In fact, if $m$ is the mass of the neutron, and $M$ the mass of the target nuclide, then the conservation of energy gives, in case of an elastic collision in the laboratory frame of reference, the following expression:

$$
E^{\prime}\left(m c^{2}+M c^{2}\right)+E\left(m c^{2}-M c^{2}\right)+E^{\prime} E=c^{2} p p^{\prime} \cos \theta,
$$

where $p=\sqrt{E\left(E+2 m c^{2}\right)} / c$ and $p^{\prime}=\sqrt{E^{\prime}\left(E^{\prime}+2 m c^{2}\right)} / c$ are the momentum of the neutron before and after the collision.
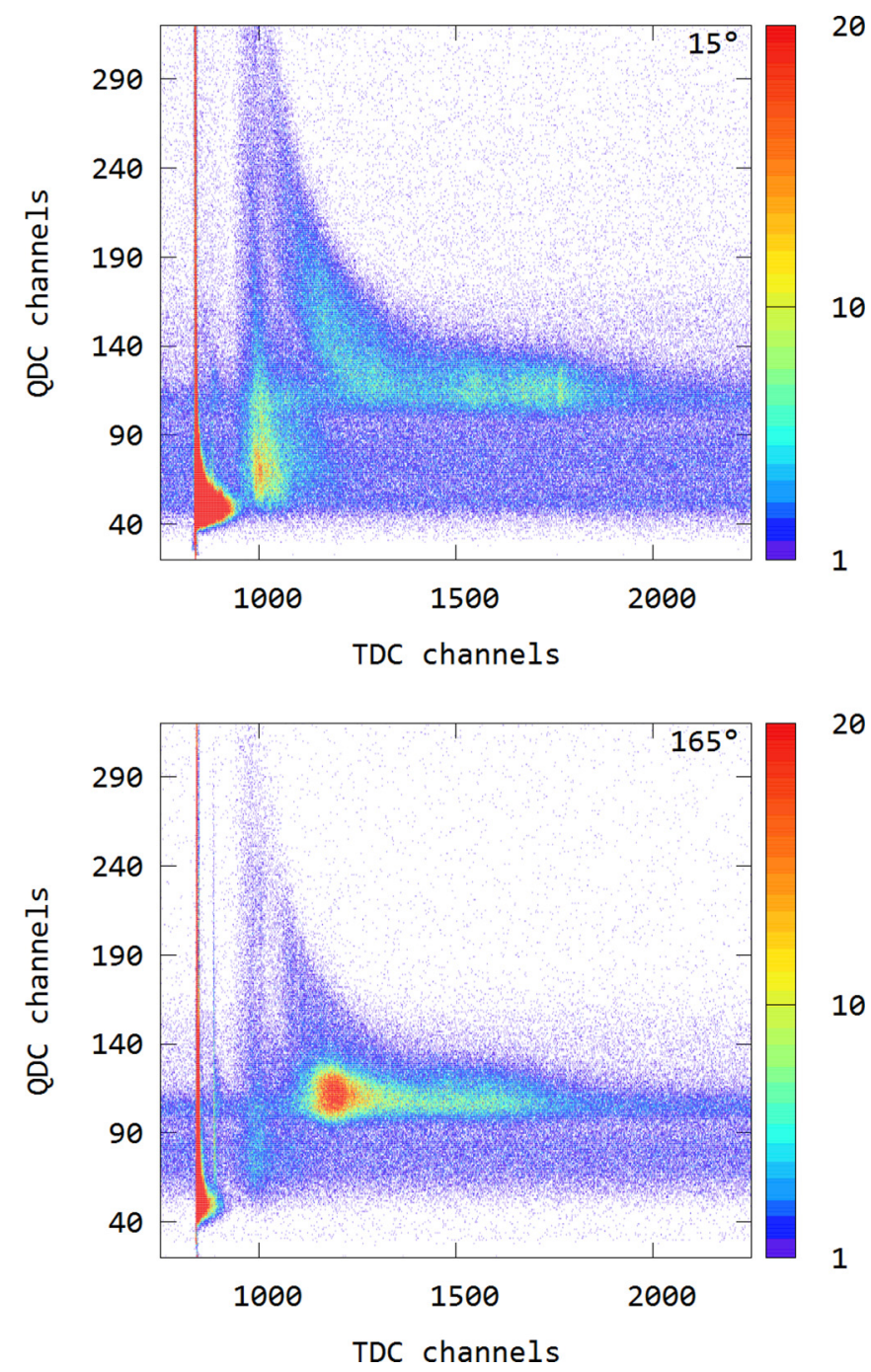

FIG. 5. Example of raw data. The two-dimensional histograms show the counts as a function of the TDC time and QDC integrated charge recorded in almost $70 \mathrm{~h}$ of beam time, with the $\mathrm{CD}_{2}$ sample as neutron scatterer, from one of the detectors at $15^{\circ}$ and one at $165^{\circ}$.

Hence, Eq. (1) states a one-to-one correspondence between the time-of-flight measured with a fixed detector and the neutron initial energy. Equation (1) however works for neutrons that scatter one time only, and if the collision happens outside the target $L$ and $L^{\prime}$ cannot be measured. Moreover, to be able to calculate $E^{\prime}$, the mass of the nuclide that was hit must be known. For this reason, one of the main objectives of the data analysis was the separation of the events due to single scattering on deuterium from those due to, for example, multiple scattering, or scattering on carbon or in air.

\section{A. Background subtraction}

In the two-dimensional histograms shown in Fig. 5, the signals of two detectors, one at $15^{\circ}$ and the other at $165^{\circ}$, were arranged according to the values given by the TDC and QDC modules, i.e., according to time and integrated charge. The absolute time of flight was obtained from the TDC values 
using the $\gamma$ flash as reference. In Fig. 5 the $\gamma$ flash is the sharp structure at the TDC channel 840 which corresponds to the time of flight of light $\left(L+L^{\prime}\right) / c$. The QDC values, proportional to the energy deposited in the active volume of the scintillator, were used to discriminate between neutron and photons. The lithium glass scintillators detect neutrons via the ${ }^{6} \mathrm{Li}(n, \alpha)^{3} \mathrm{H}$ reaction, and the relatively large $Q$ value of 4.78 MeV allows to discriminate neutron induced events from photon induced or other low-amplitude events by applying a proper gate on the charge. In the two examples in Fig. 5, the neutron events are those with QDC channel >90 and TDC channel $>1100$. The structure that can be noticed just after the TDC channel 1000 is most likely due to photons from inelastic scattering on carbon; the time-of-flight and the Monte Carlo simulations of the experiment are consistent with this explanation.

After applying proper time and charge conditions on the raw TDC-QDC matrices in order to separate the neutron events, their projections on the time axis, the t.o.f. histograms, still include a number of background events due to room return neutrons or neutrons detected after scattering in air or in the aluminium frame. As the room return background is independent from the time-of-flight, i.e., constant in time, it could be easily determined by averaging the counts in the t.o.f. intervals before the $\gamma$ flash and after the neutron burst. Figure 6 shows an example of two t.o.f. histograms after the subtraction of the time-independent component of the background.

After that, the time-dependent background component was estimated by rescaling the sample-out t.o.f. histogram with the monitor counts and then subtracting it from the $\mathrm{CD}_{2}$ and carbon measurements. The net spectra obtained after the background subtraction still had to be corrected for the multiple scattering inside the sample, and this was determined by means of a Monte Carlo model of the experiment.

\section{B. Multiple scattering}

In the model, implemented in MCNP5 [30] (see Fig. 3 for the geometry), the $\mathrm{CD}_{2}$ or graphite target and the detectors were placed in vacuum and, for the neutron source, the energy distribution measured previously in the same experimental configuration was used. The average neutron flux and the rate of the ${ }^{6} \operatorname{Li}(n, \alpha)^{3} \mathrm{H}$ reaction were tallied over the lithium glass volume as a function of the time of flight. Using the "ptrac" option it is possible to follow every history event by event, and this was used to determine the percentage of neutrons arriving at a detector after single scattering on deuterium, carbon, hydrogen (negligible), or multiple scattering in the target (see Figs. 8 and 10).

Detector by detector, the simulation results were compared with the data, finding a good overall agreement. As it is possible to notice from the two example in Figs. 7 and 9, only for short t.o.f., ca. 110-200 ns, it was not possible to reproduce the data well. However when the photon flux over the detector's sensitive volume was tallied, its shape matched this part of the spectrum, so it is possible that those unrecognised events are photons misclassified as neutrons. Anyway, this does not really pose a problem, since for neutrons scattering on deuterium the
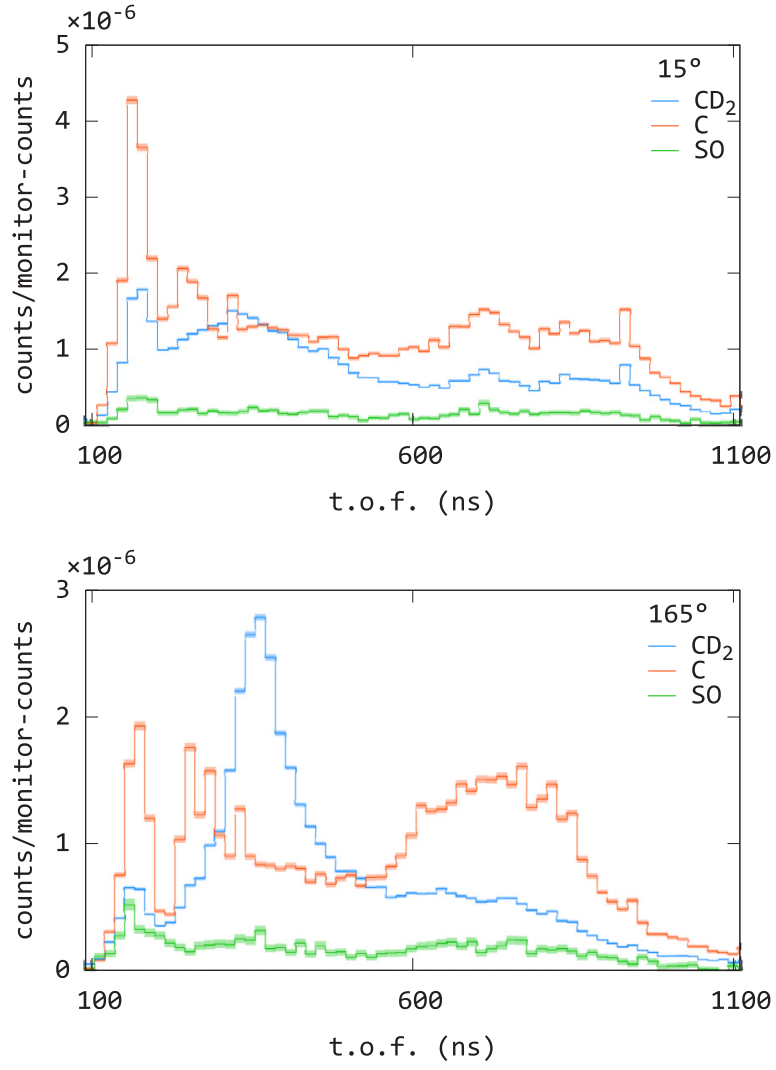

FIG. 6. Example t.o.f. histograms, one for a detector at $15^{\circ}$ and one at $165^{\circ}$, after the room background subtraction. The counts have been normalized with the monitor counts for proper comparison between the $\mathrm{CD}_{2}$, sample-out ( $\mathrm{SO}$ ) and graphite (C - carbon) measurements.

t.o.f. corresponds to incident energies of about $18 \mathrm{MeV}$ at $15^{\circ}$ and $23 \mathrm{MeV}$ at $165^{\circ}$, well beyond the range of interest.

For these simulations, all cross section libraries were from ENDF/B-VII. No other library was considered at this stage because the aim was to estimate the multiple scattering in the target, not to appraise different evaluations.

Without a measurement of the neutron flux model and experiment cannot be compared directly. Hence, the MCNP results were rescaled to the data by a constant factor, which was found by minimizing the difference between data and simulations with the linear least square method applied in the t.o.f. interval from 450 to $750 \mathrm{~ns}$. This interval was defined in the attempt to find a region in the energy distributions with good statistics and small contribution of the multiple scattering component when compared to the single scattering.

In principle, the rescaling factor depends only on the incident flux, and thus can serve as parameter for a consistency check: if the detectors are all equivalent, the rescaling coefficient must be the same for all of them, independently from their position or the sample in place. It was computed assuming that the ratio between the counts at a given t.o.f. in the experimental histograms normalized by the monitor counts [DATA(t.o.f.)] and the ${ }^{6} \operatorname{Li}(n, \alpha)$ events scored by MCNP for the same t.o.f. [MCNP(t.o.f.)] was constant, by the linear regression of the relation: DATA(t.o.f.) $=b \times \operatorname{MCNP}($ t.o.f.). 

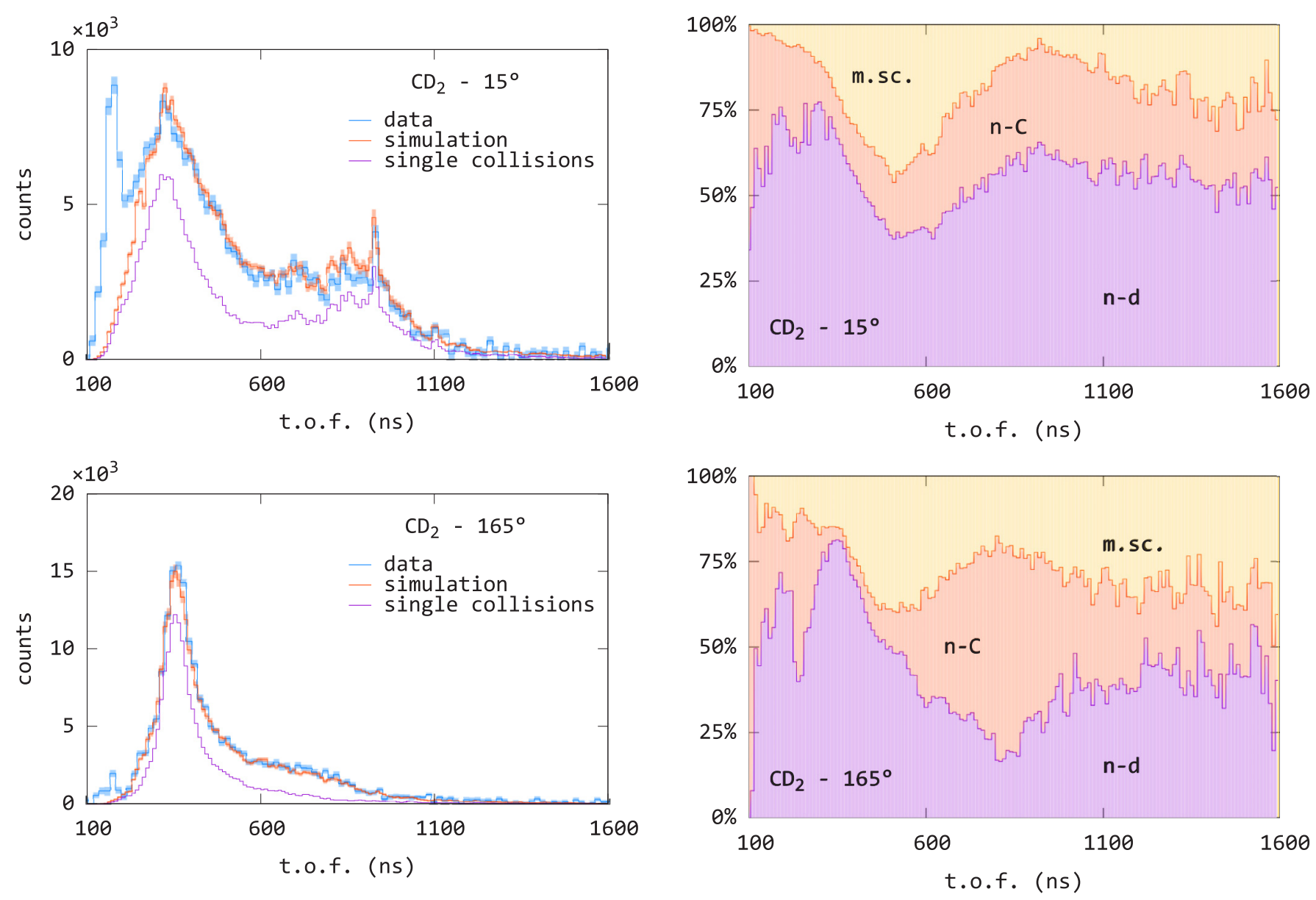

FIG. 7. Experimental $\mathrm{CD}_{2}$ t.o.f. histograms ("data”), at $15^{\circ}$ and $165^{\circ}$, after the subtraction of the contribution of scattering in air, compared with the simulated ${ }^{6} \operatorname{Li}(n, \alpha)$ reaction rate tallied in the detector sensitive volume ("simulation"). The "single collisions" lines are the simulation results when only neutrons arriving after a single collision on deuterium are considered.

The results of the regression for the parameter $b$, obtained for each detector and for each run with a different target (see Fig. 11), were consistent with each other. The arithmetic average of all values was therefore used to rescale the MCNP simulation to the data.

To investigate the accuracy of the model, the sample-out measurement was also reproduced (see Fig. 12). In this case, only the target holder was modelled and a cylindrical volume of air was positioned around the beam axis while the detectors were still in vacuum. The outcome confirmed that the t.o.f. dependent background component, as measured in the sampleout measurement, mainly results from neutrons scattering once or twice in air before being detected.

It was also possible to prove that adding further details to the model (the aluminium frame, air in the whole room, the concrete walls) did not significantly change the results. Including the detector supporting structure, for example, led to a systematic increase in the number of ${ }^{6} \mathrm{Li}(n, \alpha)^{3} \mathrm{H}$ events tallied as function of the t.o.f. over the detectors' sensitive volume. At $2 \mathrm{MeV}$ of neutron incident energy this increase amounted to $1 \%$ of the value obtained with the original

FIG. 8. Contribution of multiple scattering in the target ("m.sc."), single scattering on deuterium (" $n-d$ ") or on carbon (" $n-C$ "), expressed in percentage of number of events as a function of the time of flight.

model, at $500 \mathrm{keV}$ it was $4 \%$, and at $200 \mathrm{keV}$ it was $5 \%$. This contribution however was lower than the relative statistical uncertainties of the simulation, which ranged from $2 \%$ at $2 \mathrm{MeV}$ to $7 \%$ at $200 \mathrm{keV}$. Also, it was regarded as negligible compared to the uncertainties on the experimental t.o.f. histograms, which went from $4 \%$ at $2 \mathrm{MeV}$ to $20 \%$ at $200 \mathrm{keV}$. Modelling the concrete walls that delimited the experimental hall and filling the empty space with air caused a difference that fluctuated between $-2 \%$ and $2 \%$ in the range from 1 to $2 \mathrm{MeV}$, and between $-5 \%$ and $5 \%$ in the range from $200 \mathrm{keV}$ to $500 \mathrm{keV}$. The combined effects of considering both the aluminium frame and the air-filled room varied between $-2 \%$ and $2 \%$ at $1-2 \mathrm{MeV},-2 \%$ and $4 \%$ at $0.5-1 \mathrm{MeV},-5 \%$ and $7 \%$ at $0.2-0.5 \mathrm{MeV}$, always lying within the limits of the uncertainties.

\section{Scattering on deuterium}

Since the simulations allowed to assess the fraction of detected events happening after multiple scattering, the data were reduced to have only events due to single collisions in the target. At this point, knowing the areal density of both the $\mathrm{CD}_{2}$ and graphite samples, the graphite t.o.f. spectra 

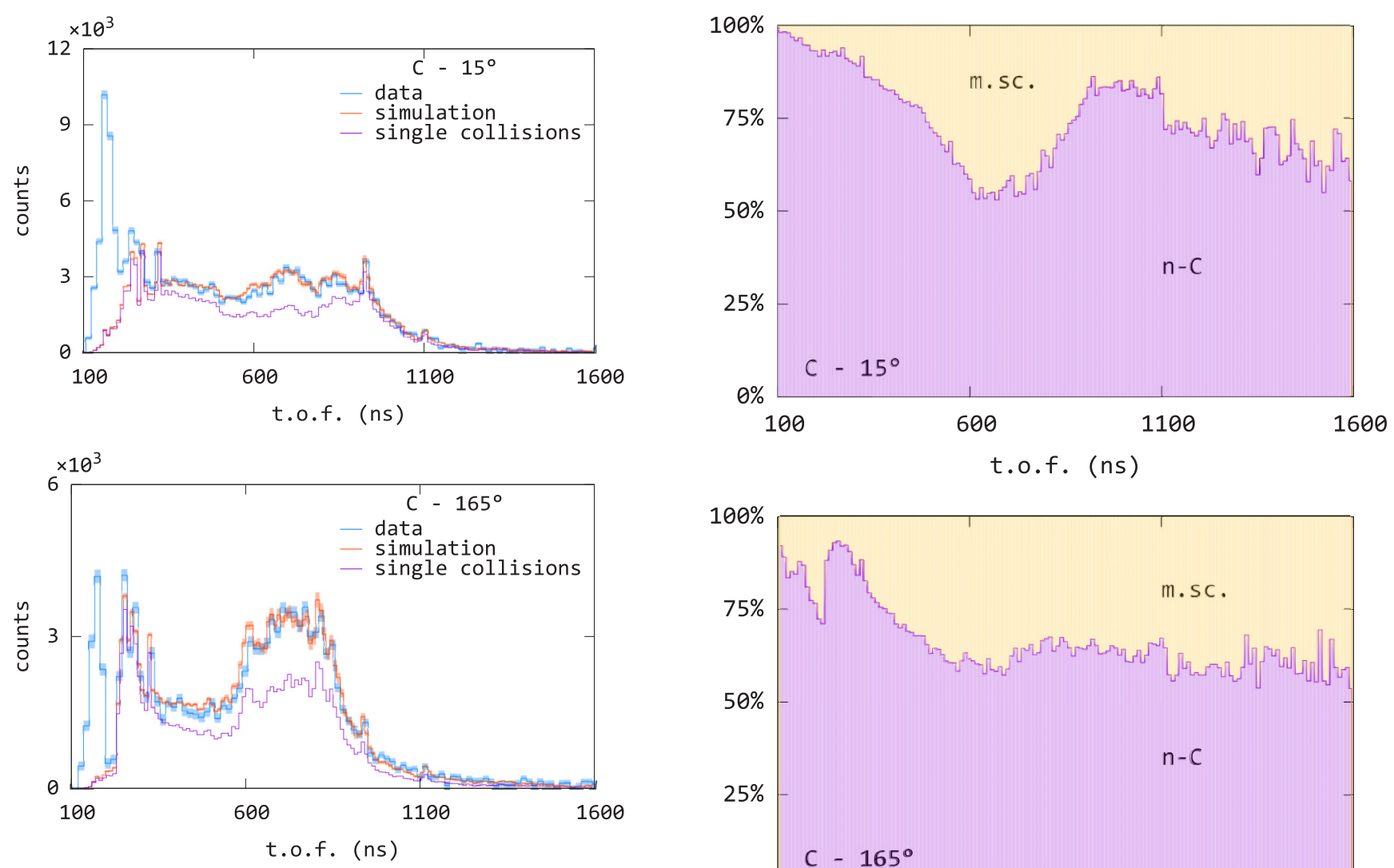

FIG. 9. Same as Fig. 7 but for the carbon data. In this case the "single collisions" lines indicate the single collision on carbon.

were multiplied by the ratio of the masses per unit area to determine and subtract the contribution of carbon from the $\mathrm{CD}_{2}$ measurement. The spectra for single scattering on deuterium obtained after the subtraction are shown in Fig. 13, with the time of flight converted in neutron incident energy. The spectra of the detectors at the same angle are very similar and, therefore, the counts were summed together without further corrections.

The two total histograms at $15^{\circ}$ and $165^{\circ}$ cannot be directly compared because of the detection efficiency, which does not depend on the incident energy. Assuming all detectors are equivalent, the efficiency depends on the angle in the sense that for the same initial (incident) energy, neutrons scattered at $15^{\circ}$ have a different final energy from those arriving at $165^{\circ}$, so a different probability of being detected.

The detection efficiency was determined analyzing the results of the simulation with the $\mathrm{CD}_{2}$ target, the ptrac file, a second time. Considering only the histories including one single $n$ - $d$ scattering event, the number of ${ }^{6} \mathrm{Li}(n, t)^{4} \mathrm{He}$ events relative to the number of incoming neutrons and thus the detection efficiency were determined as function of the neutron energy $E$ as it is before colliding with the target. This method was used in order to be able to take automatically into account the full geometry of the experiment and the changes in the neutron energy after every collision in the target and in the detectors. Figure 14 shows the efficiency $\epsilon(E)$ at the two scattering angles $165^{\circ}$ and $15^{\circ}$ as function of the incident energy on deuterium.

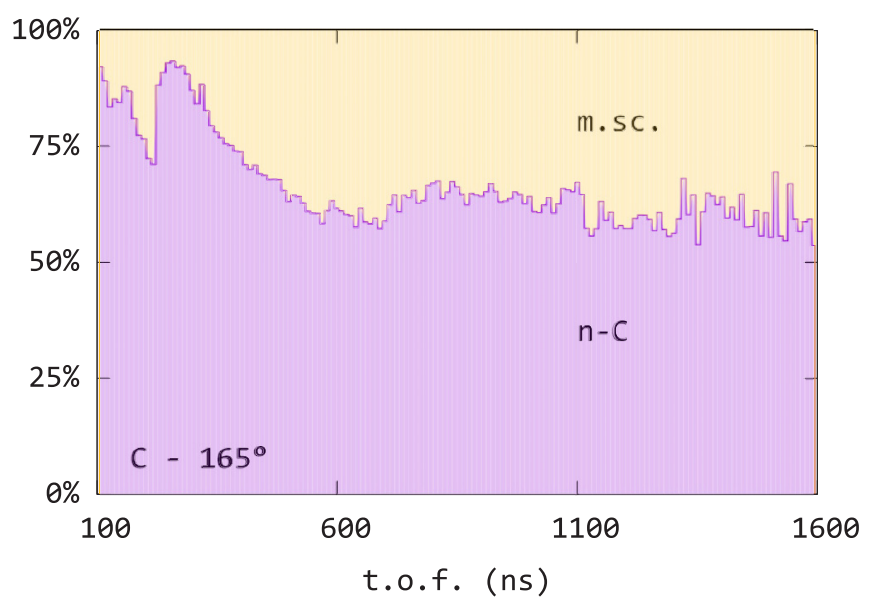

FIG. 10. Same as Fig. 8 but for the carbon data.

Finally, the ratio between $165^{\circ}$ and $15^{\circ}$ was computed in function of the incident energy $E$ by

$$
\frac{\left.\frac{d \sigma}{d \Omega}(E)\right|_{165^{\circ}}}{\left.\frac{d \sigma}{d \Omega}(E)\right|_{15^{\circ}}}=\frac{\left.\left.n_{\text {single }}(E)\right|_{165^{\circ}} \varepsilon(E)\right|_{15^{\circ}}}{\left.\left.n_{\text {single }}(E)\right|_{15^{\circ}} \varepsilon(E)\right|_{165^{\circ}}},
$$

where $n_{\text {single }}$ is the number of detected events due to single scattering on deuterium.

\section{RESULTS}

Figure 15 shows the experimental results of $\left.\frac{d \sigma}{d \Omega}\right|_{165^{\circ}} /\left.\frac{d \sigma}{d \Omega}\right|_{15^{\circ}}$, compared with the evaluated libraries, the theoretical calculations of Canton et al. [11] and of Golak et al. [15,31]. Because of the low counting statistics, especially at $165^{\circ}$, and thus of the large uncertainties, it was not possible to extend the results below $200 \mathrm{keV}$. Above $2 \mathrm{MeV}$, the data cannot be fully trusted because the detectors response was not fully modelled (see Figs. 7, 9, and 12 for t.o.f. $<300 \mathrm{~ns}$ ). For example, the ${ }^{6} \mathrm{Li}\left(n, n^{\prime} d\right)^{4} \mathrm{He}$ reaction, which has a negative $Q$ value of $-1.47 \mathrm{MeV}$, was not included in the simulations for the determination of the efficiency. This does not affect the data of the detectors at backward angles, because neutrons with initial energy of $2 \mathrm{MeV}$ have a kinetic energy of $230 \mathrm{keV}$ after the scattering at $165^{\circ}$; but it could have some impact for the data of detectors at $15^{\circ}$. 


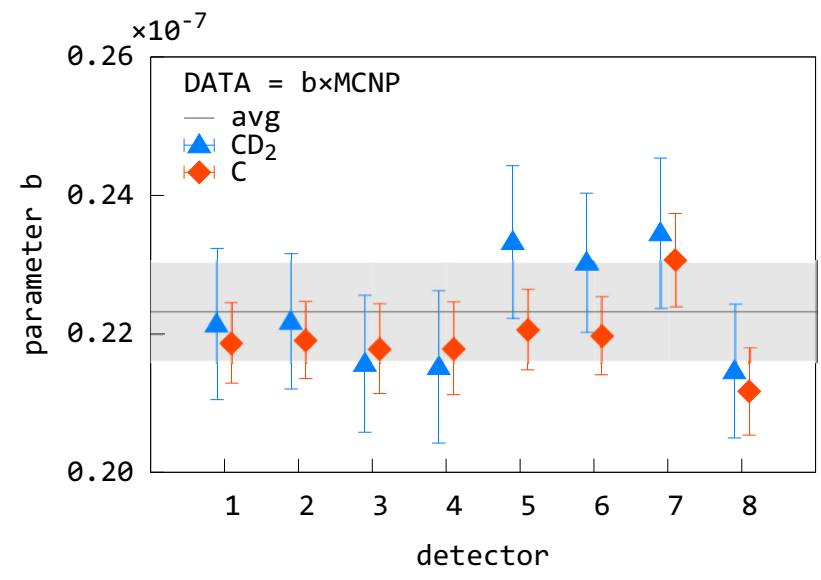

FIG. 11. Rescaling factor for the MCNP results obtained for each detector (detectors at $15^{\circ}$ : numbers $3,4,7$, and 8 at $165^{\circ}: 1,2,5$, and 6 ), for the $\mathrm{CD}_{2}$ and graphite (C) runs. The parameter $b$ has an order of magnitude of $10^{-7}$ because the data were normalized by the monitor counts. The arithmetic average (avg) of all values is what was used to rescale the simulations to the data in Figs. 7, 9, and 12.

In general, the data suggest that in the energy range from 350 to $600 \mathrm{keV}$ the $n-d$ scattering angular distribution is either more isotropic or more backward peaked than how it is reported in the evaluated nuclear libraries. Overall, the data are compatible with the theoretical calculations of Canton
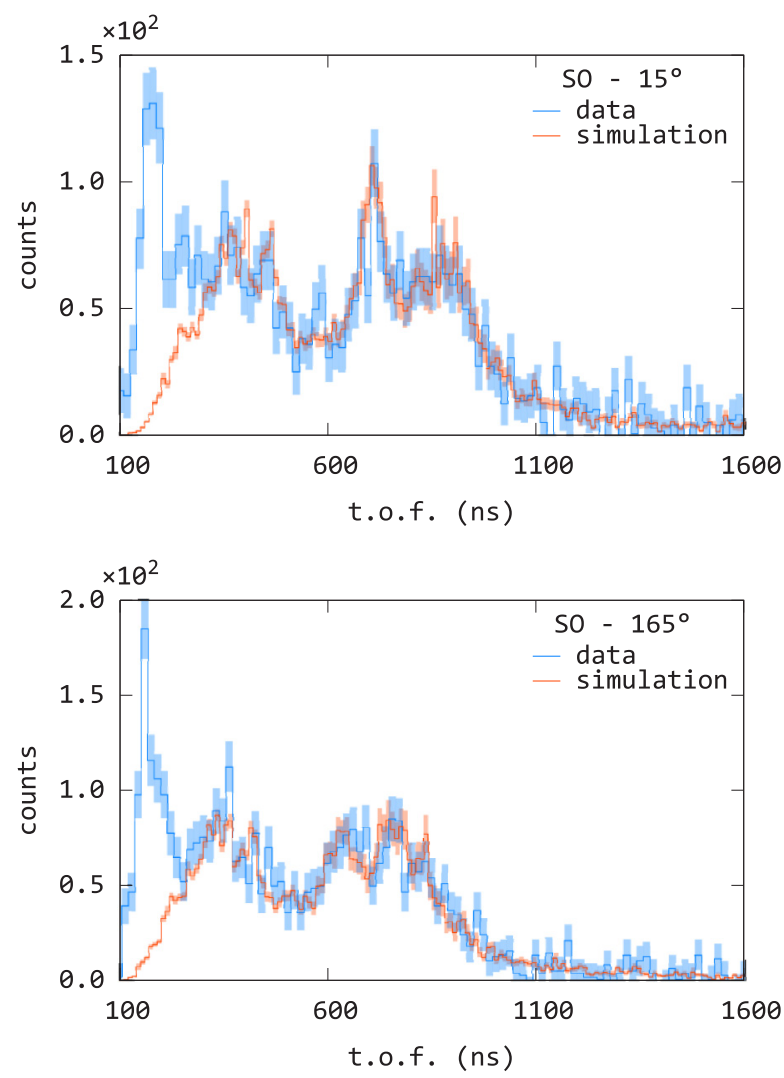

FIG. 12. Experimental t.o.f. histograms ("data") for the sampleout run, at $15^{\circ}$ and $165^{\circ}$, compared to the simulated ${ }^{6} \mathrm{Li}(n, \alpha)$ reaction rate tallied in the glass volume ("simulation").
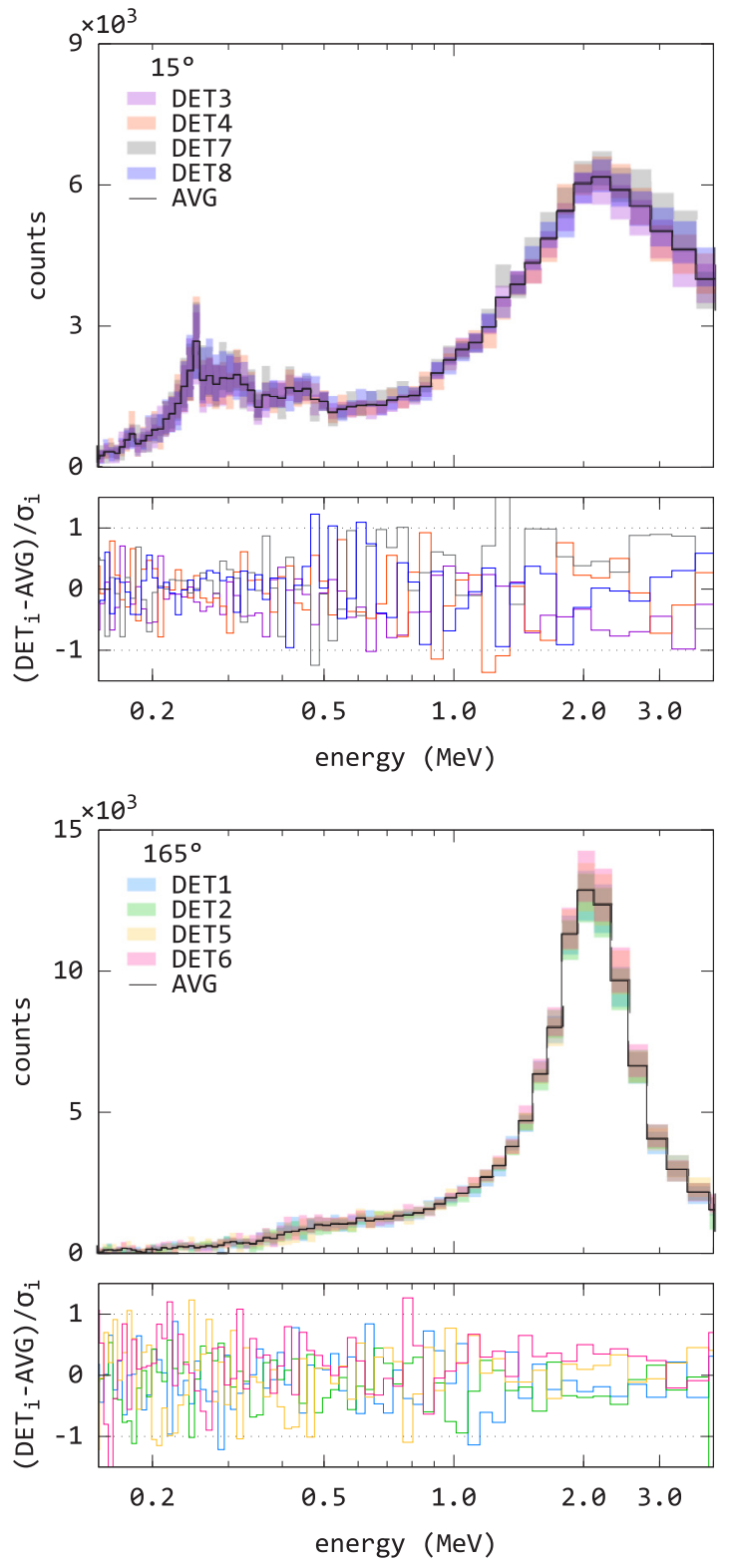

FIG. 13. Single scattering on deuterium events at $15^{\circ}$ and $165^{\circ}$, for every detector alone $\left(\operatorname{DET}_{i}(E)\right)$, and their average $(\operatorname{AVG}(E))$, as a function of the incident neutron energy $E$. The deviation from the average, expressed as $\left(\operatorname{DET}_{i}(E)-\operatorname{AVG}(E)\right) / \sigma_{\mathrm{DET}_{i}}(E)$, where $\sigma$ is the uncertainty on the measured events, is also plotted for each detector.

et al. and the recent EFT based one of Golak et al., and the evaluated libraries CENDL-3.1 and JEFF-3.2. Also JENDL4.0 is compatible, but the dip at $600 \mathrm{keV}$ does not seem to be very physical. ENDF/B-VII. 1 is in agreement with the data up to $700 \mathrm{keV}$, slightly below the measurements for energies below $500 \mathrm{keV}$. From $700 \mathrm{keV}$ to $1.7 \mathrm{MeV}$ the ratio $165^{\circ}$ over $15^{\circ}$ is higher that what found experimentally. ROSFOND-2010 is consistent only with the data below $490 \mathrm{keV}$ and BROND2.2 exhibits the largest deviations, but those two libraries are also the oldest evaluations among those presented.

To understand to what degree the comparison between the experimental results and the different options for the angular distribution of $n$ - $d$ scattering could be affected by the method 


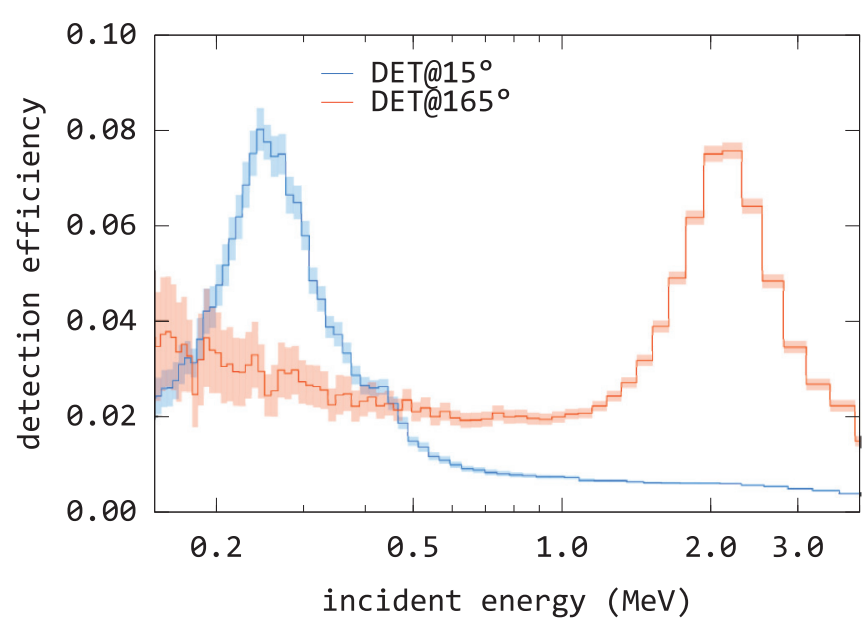

FIG. 14. Calculated detection efficiency for detectors at $15^{\circ}$ and at $165^{\circ}$ as a function of the neutron energy incident on deuterium.

of data analysis, we provide in Fig. 16 a direct comparison of the raw experimental data with a full simulation of the setup. This has the advantage that no approximations are made to account for the detector response. The data are shown with the background from the sample-out run subtracted. The calculation uses the full specification of the neutron beam, the sample, the detectors and the frame that holds them. The data used for each nucleus were kept the same (ENDF/B-VII) except for the data for deuterium. The region of normalization is for time of flights between 450 and $750 \mathrm{~ns}$. To better visualize the differences the ratio between the calculations and the experimental data are plotted in Fig. 17.

In the energy range from $200 \mathrm{keV}$ to $2 \mathrm{MeV}$ the calculations for $n-d$ scattering that are in best agreement with the experimental data are by Canton et al. and those included in JENDL-4.0. At $15^{\circ}$ the cross section data calculated by Canton et al. show smaller deviations from the experiment. For both

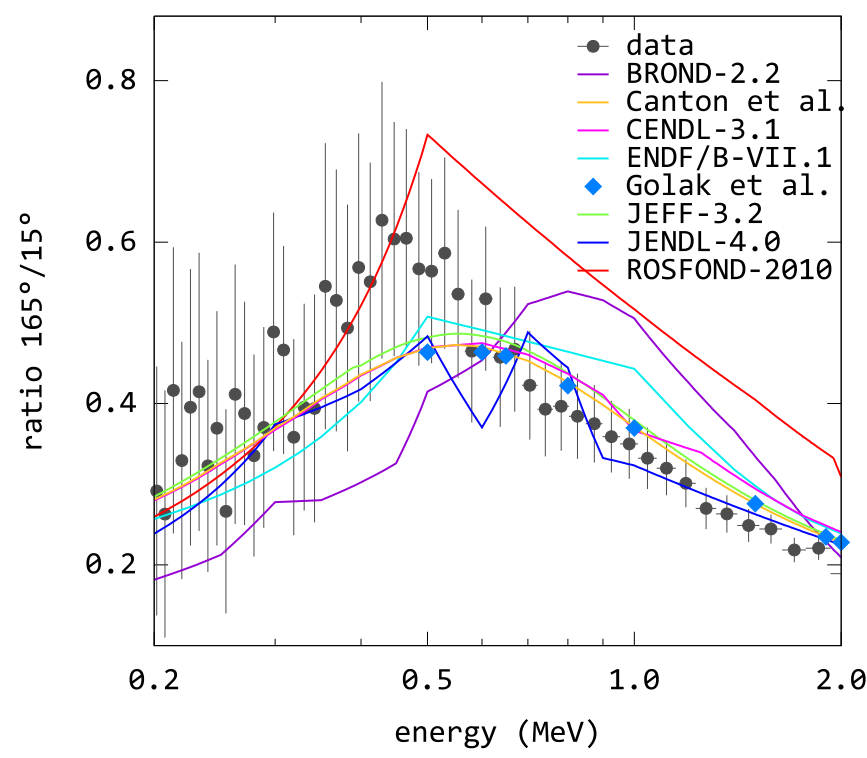

FIG. 15. Comparison of the ratio $\left.\frac{d \sigma}{d \Omega}\right|_{165^{\circ}} /\left.\frac{d \sigma}{d \Omega}\right|_{15^{\circ}}$ : experimental results versus the main evaluated libraries and the calculations of Canton et al.
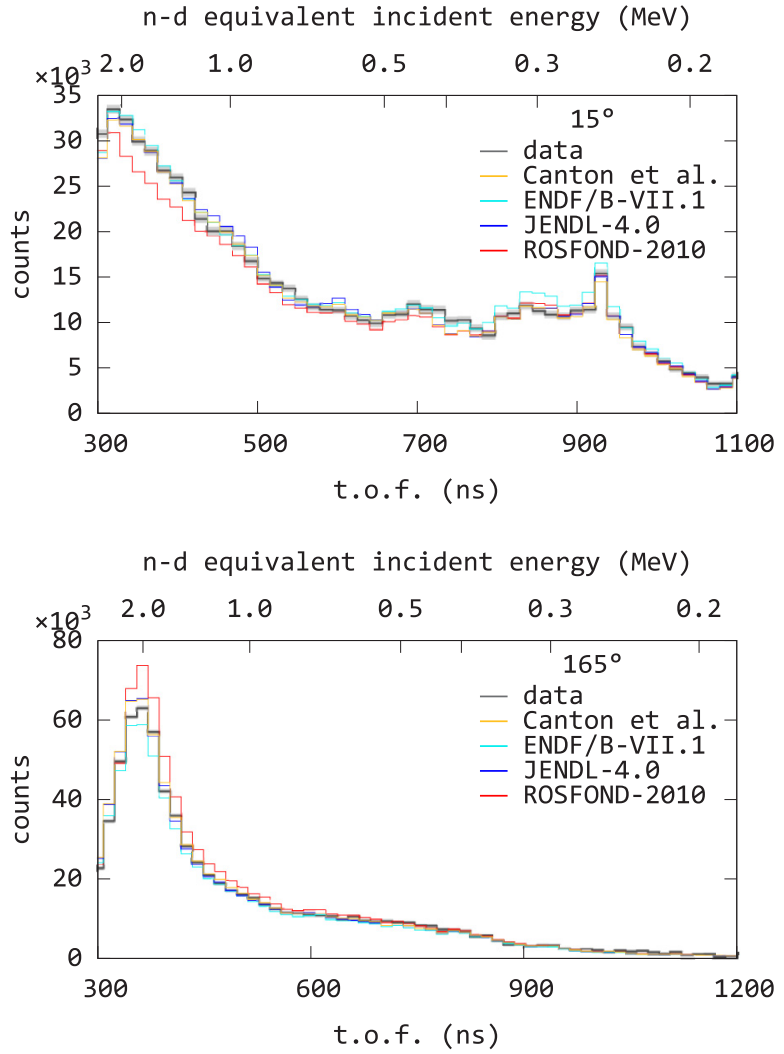

FIG. 16. Comparison the experimental t.o.f. spectra with the MCNP5 simulations obtained after using several different libraries for the deuterium cross section tables. These spectra are the sum of the counts coming from all detectors at same angle.

libraries, the difference with the data remains within $10 \%$ in the entire range at $15^{\circ}$ and above $300 \mathrm{keV}$ at $165^{\circ}$. Below $300 \mathrm{keV}$, however, the low statistic at $165^{\circ}$ results in large fluctuations. At $15^{\circ}$ for t.o.f. from 720 to $780 \mathrm{~ns}$, i.e., from about $355 \mathrm{keV}$ to $420 \mathrm{keV}$ of neutron energy, there is a clear dip where all four simulations display a similar trend. A similar feature, but less pronounced, is also found at $165^{\circ}$ in the same t.o.f. interval. This suggests that this discrepancy is not due to the deuterium cross section since for scattering on deuterium the same time-of-flight interval corresponds to very different detected energies. If this were an artifact of the simulation, then in Fig. 15 the experimental data for the differential cross sections ratio of $165^{\circ}$ to $15^{\circ}$ would be reduced by about $5 \%$ for incident neutrons with energies between $355 \mathrm{keV}$ and $420 \mathrm{keV}$. For the simulations using the ENDF/B-VII.1 evaluated library the ratio "MCNP/data" remains within $15 \%$ from the data everywhere at $165^{\circ}$, while at $15^{\circ}$ it is so only for energies above $355 \mathrm{keV}$ (t.o.f. $<780 \mathrm{~ns}$ ). If only the values that were not used for the normalization are considered, then it means that ENDF/BVII.1 tends to overestimate the scattering at $15^{\circ}$ for energies below $355 \mathrm{keV}$. ROSFOND-2010 is compatible with the data only at low energies: below $530 \mathrm{keV}$ (t.o.f. $>700 \mathrm{~ns}$ ) at $165^{\circ}$ and below $350 \mathrm{keV}$ (t.o.f. $>790 \mathrm{~ns}$ ) at $15^{\circ}$. In the rest of the range, the simulation overestimates the number of events at the backward angles and underestimates them at forward angles, which implies that the angular distribution should be less backward peaked. For the calculations by Golak et al. no 

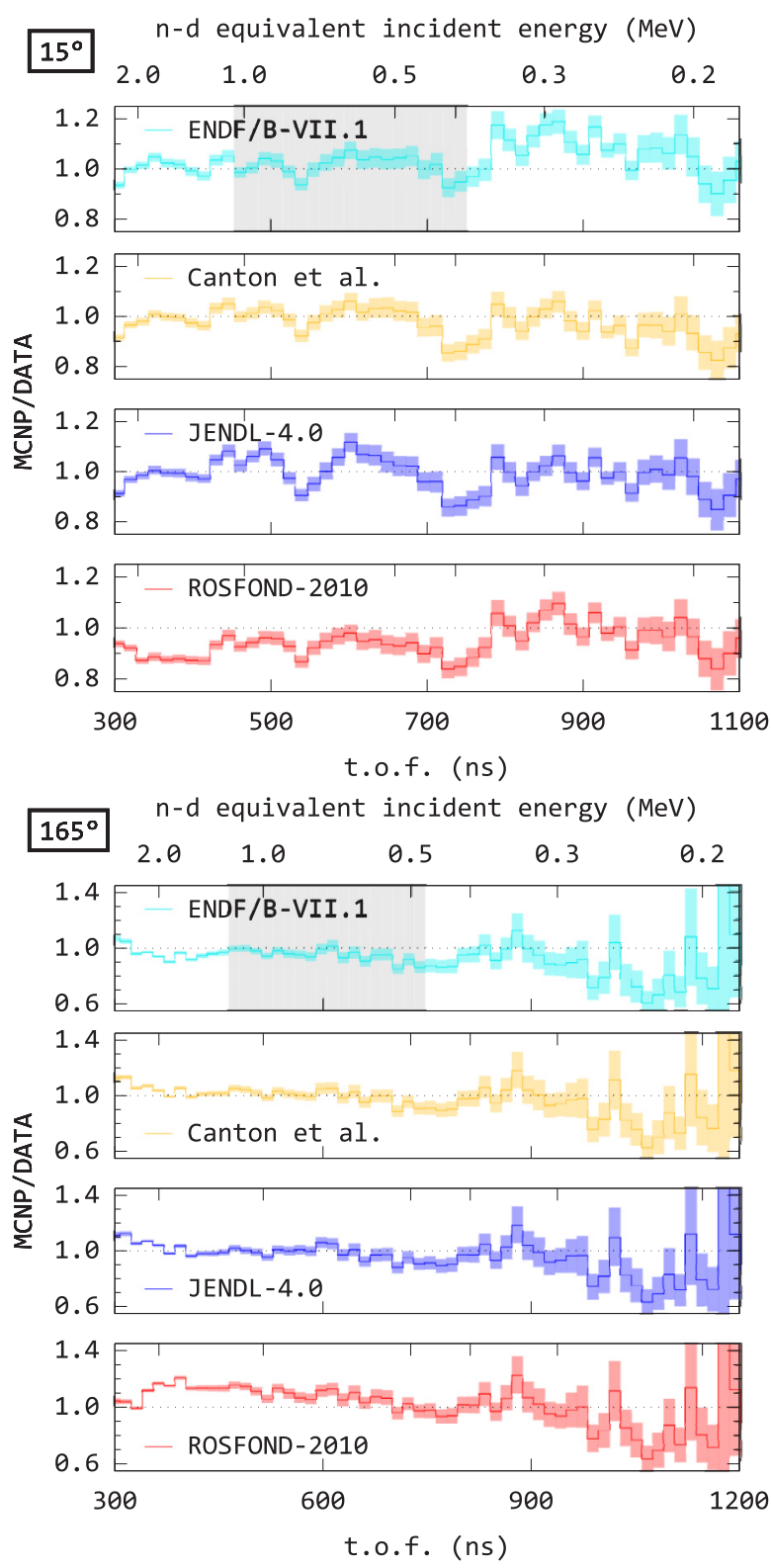

FIG. 17. Ratio between the MCNP and the experimental t.o.f. histograms shown in Fig. 16. The grey area represent the t.o.f. interval considered when computing the rescaling factor for the comparison between data and simulation.

MCNP compatible file (ACE file) was available allowing a similar comparison. The strong similarity with Canton et al. in Fig. 15 suggests it would perform similarly in Figs. 16 and 17 as well.

\section{v. CONCLUSIONS}

A new experiment for the investigation of the $n$ - $d$ scattering angular distribution was performed at the neutron t.o.f. facility nELBE. The measurement, carried out using a highly enriched $\mathrm{CD}_{2}$ sample, was complemented with two additional runs, one with graphite and the other with no sample. The three runs were realized so to be able to discriminate between $n$ - $d$ scattering and $n$-carbon scattering or background events. Extensive MCNP simulations with a realistic experimental geometry and neutron flux were used to subtract the multiple scattering contribution and to determine the efficiency of the detectors.

The ratio of the differential cross section at $15^{\circ}$ and $165^{\circ}$ was determined, and the results cover the neutron energy range from $200 \mathrm{keV}$ to $2 \mathrm{MeV}$. The experimental data are in agreement with the theoretical calculations performed by Canton et al. and Golak et al., who obtained highly compatible predictions for the $n-d$ scattering differential cross section using two different formulations of the nuclear potential. The comparison with the evaluated nuclear data libraries indicates CENDL-3.1, JEFF-3.2 and JENDL-4.0 as the evaluations that best describe the asymmetry of the $n-d$ scattering. In the energy range from 350 and $600 \mathrm{keV}$, however, the experimental backward to forward ratio is higher than that reported in the libraries. This suggests that the angular distributions should be either more isotropic or more backward peaked. ENDF/B-VII.1 predicts a higher backward to forward ratio than that actually measured for energies above $700 \mathrm{keV}$, while below $500 \mathrm{keV}$ it is slightly lower. ROSFOND-2010 and BROND-2.2 are based on old evaluations and also have the worst compatibility with the experimental data.

\section{ACKNOWLEDGMENTS}

The authors are grateful to all colleagues from the HZDR Institute of Radiation Physics for their support before, during and after the measurement, and in particular to Mirco Dietz, Marcel Grieger, Toni Kögler, Konrad Schmidt, Ronald Schwengner, Marcell Peter Takacs, and Louis Wagner. They wish to thank Ken Kozier, Raghu Rao, and Dan Roubtsov for the procurement of the $\mathrm{CD}_{2}$ target and the support that helped shape the experiment. They would also like to express their appreciation to Luciano Canton for sharing his insights on the subject and the valuable discussion and to Henryk Witała for kindly providing us with the theoretical cross sections. This work was partly supported by the European Commission within the Seventh Framework Programme through CHANDA (Grant Agreement No. 605203).
[1] J. Atanackovic, A. Yonkeu, J. Dubeau, S. H. Witharana, and N. Priest, Appl. Rad. Isotopes 99, 122 (2015).

[2] H. Wang and A. Enqvist, Nucl. Instrum. Methods Phys. Res. A 804, 167 (2015).

[3] OECD Nuclear Energy Agency, Nuclear Data High-Priority Request List, available at http://www.oecd-nea.org/dbdata/hprl/.
[4] N. Otuka, E. Dupont, V. Semkova, B. Pritychenko, A. I. Blokhin, M. Aikawa, S. Babykina, M. Bossant, G. Chen, S. Dunaeva, R. A. Forrest, T. Fukahori, N. Furutachi, S. Ganesan, Z. Ge, O. O. Gritzay, M. Herman, S. Hlavač, K. Katō, B. Lalremruata, Y. O. Lee, A. Makinaga, K. Matsumoto, M. Mikhaylyukova, G. Pikulina, V. G. Pronyaev, A. Saxena, O. Schwerer, S. P. 
Simakov, N. Soppera, R. Suzuki, S. Takács, X. Tao, S. Taova, F. Tárkányi, V. V. Varlamov, J. Wang, S. C. Yang, V. Zerkin, and Y. Zhuang, Nucl. Data Sheets 120, 272 (2014).

[5] L. W. Townsend, Neutron-deuterium cross section evaluation, Final Technical Report AECL Purchase Order 217739 (Atomic Energy of Canada Limited, 2006).

[6] R. D. Mosteller, J. M. Campbell, and R. C. Little, 2005 Annual Meeting of the American Nuclear Society (American Nuclear Society, San Diego, CA, 2005), pp. 669-671.

[7] K. S. Kozier, PHYSOR 2006 Topical Meeting: Advances in Nuclear Analysis and Simulation (American Nuclear Society, Vancouver, BC, 2006), pp. 2123-2132.

[8] Cross Section Evaluation Working Group, ENDF/B-VI Summary Documentation, Report No. BNL-NCS-17541 (ENDF201) (Brookhaven National Laboratory, 1991), edited by P. F. Rose.

[9] K. Shibata, T. Kawano, T. Nakagawa, O. Iwamoto, J. Katakura, T. Fukahori, S. Chiba, A. Hasegawa, T. Murata, H. Matsunobu, T. Ohsawa, Y. Nakajima, T. Yoshida, A. Zukeran, M. Kawai, M. Baba, M. Ishikawa, T. Asami, T. Watanabe, Y. Watanabe, M. Igashira, N. Yamamuro, H. Kitazawa, N. Yamano, and H. Takano, J. Nucl. Sci. Technol. 39, 1125 (2002).

[10] The JEFF-3.1 Nuclear Data Library, JEFF Report 21 (OECD Nuclear Energy Agency, 2006), edited by A. Koning, R. Forrest, M. Kellett, R. Mills, H. Henriksson, and Y. Rugama.

[11] L. Canton, W. Schadow, and J. Haidenbauer, Eur. Phys. J. A 14, 225 (2002).

[12] J. P. Svenne, L. Canton, K. Kozier, and L. Townsend, International Conference on Nuclear Data for Science and Technology (Nice, 2007), Vol. 1, pp. 243-246.

[13] M. B. Chadwick, P. Obložinský, M. Herman, N. M. Greene, R. D. McKnight, D. L. Smith, P. G. Young, R. E. MacFarlane, G. M. Hale, S. C. Frankle, A. C. Kahler, T. Kawano, R. C. Little, D. G. Madland, P. Moller, R. D. Mosteller, P. R. Page, P. Talou, H. Trellue, M. C. White, W. B. Wilson, R. Arcilla, C. L. Dunford, S. F. Mughabghab, B. Pritychenko, D. Rochman, A. A. Sonzogni, C. R. Lubitz, T. H. Trumbull, J. P. Weinman, D. A. Brown, D. E. Cullen, D. P. Heinrichs, D. P. McNabb, H. Derrien, M. E. Dunn, N. M. Larson, L. C. Leal, A. D. Carlson, R. C. Block, J. B. Briggs, E. T. Cheng, H. C. Huria, M. L. Zerkle, K. S. Kozier, A. Courcelle, V. Pronyaev, and S. C. van der Marck, Nucl. Data Sheets 107, 2931 (2006), Evaluated Nuclear Data File ENDF/B-VII.0.

[14] L. E. Marcucci, A. Kievsky, L. Girlanda, S. Rosati, and M. Viviani, Phys. Rev. C 80, 034003 (2009).

[15] J. Golak, R. Skibiński, K. Topolnicki, H. Witała, E. Epelbaum, H. Krebs, H. Kamada, U. G. Meißner, V. Bernard, P. Maris, J. Vary, S. Binder, A. Calci, K. Hebeler, J. Langhammer, R. Roth, A. Nogga, S. Liebig, and D. Minossi, Eur. Phys. J. A 50, 177 (2014).

[16] S. Binder, A. Calci, E. Epelbaum, R. J. Furnstahl, J. Golak, K. Hebeler, H. Kamada, H. Krebs, J. Langhammer, S. Liebig, P. Maris, Ulf G. Meißner, D. Minossi, A. Nogga, H. Potter, R. Roth, R. Skibiński, K. Topolnicki, J. P. Vary, and H. Witała, Phys. Rev. C 93, 044002 (2016).

[17] A. I. Blokhin, B. I. Fursov, A. V. Ignatyuk, V. N. Koshcheev, E. V. Kulikov, B. D. Kuzminov, V. N. Manokhin, and M. N. Nikolaev, International Conference on Nuclear Datafor Science and Technology (Gatlinburg, TN, 1994), Vol. 2, pp. 695-698.
[18] Z. G. Ge, Z. X. Zhao, H. H. Xia, Y. X. Zhuang, T. J. Liu, J. S. Zhang, and H. C. Wu, J. Korean Phys. Soc. 59, 1052 (2011).

[19] OECD Nuclear Energy Agency, "JEFF-3.2 Evaluated Data Library", available at https://www.oecd-nea.org/dbforms/ data/eva/evatapes/jeff_32/

[20] K. Shibata, O. Iwamoto, T. Nakagawa, N. Iwamoto, A. Ichihara, S. Kunieda, S. Chiba, K. Furutaka, N. Otuka, T. Ohsawa, T. Murata, H. Matsunobu, A. Zukeran, S. Kamada, and J. I. Katakura, J. Nucl. Sci. Technol. 48, 1 (2011).

[21] M. B. Chadwick, M. Herman, P. Obložinský, M. E. Dunn, Y. Danon, A. C. Kahler, D. L. Smith, B. Pritychenko, G. Arbanas, R. Arcilla, R. Brewer, D. A. Brown, R. Capote, A. D. Carlson, Y. S. Cho, H. Derrien, K. Guber, G. M. Hale, S. Hoblit, S. Holloway, T. D. Johnson, T. Kawano, B. C. Kiedrowski, H. Kim, S. Kunieda, N. M. Larson, L. Leal, J. P. Lestone, R. C. Little, E. A. McCutchan, R. E. MacFarlane, M. MacInnes, C. M. Mattoon, R. D. McKnight, S. F. Mughabghab, G. P. A. Nobre, G. Palmiotti, A. Palumbo, M. T. Pigni, V. G. Pronyaev, R. O. Sayer, A. A. Sonzogni, N. C. Summers, P. Talou, I. J. Thompson, A. Trkov, R. L. Vogt, S. C. van der Marck, A. Wallner, M. C. White, D. Wiarda, and P. G. Young, Nucl. Data Sheets 112, 2887 (2011), special issue on ENDF/B-VII.1 Library .

[22] Institute of Physics and Power Engineering, "ROSFOND2010 library", available at http://www.ippe.ru/podr/abbn/libr/ rosfond.php

[23] E. Altstadt, C. Beckert, H. Freiesleben, V. Galindo, E. Grosse, A. R. Junghans, J. Klug, B. Naumann, S. Schneider, R. Schlenk, A. Wagner, and F. P. Weiss, Ann. Nucl. Energy 34, 36 (2007).

[24] J. Klug, E. Altstadt, C. Beckert, R. Beyer, H. Freiesleben, V. Galindo, E. Grosse, A. R. Junghans, D. Légrády, B. Naumann, K. Noack, G. Rusev, K. D. Schilling, R. Schlenk, S. Schneider, A. Wagner, and F. P. Weiss, Nucl. Instrum. Methods Phys. Res. A 577, 641 (2007).

[25] N. Nankov, A. J. M. Plompen, S. Kopecky, K. S. Kozier, D. Roubtsov, R. Rao, R. Beyer, E. Grosse, R. Hannaske, A. R. Junghans, R. Massarczyk, R. Schwengner, D. Yakorev, A. Wagner, M. Stanoiu, L. Canton, R. Nolte, S. Röttger, J. Beyer, and J. Svenne, Nucl. Data Sheets 119, 98 (2014).

[26] F. Gabriel, P. Gippner, E. Grosse, D. Janssen, P. Michel, H. Prade, A. Schamlott, W. Seidel, A. Wolf, and R. Wünsch, Nucl. Instrum. Methods Phys. Res. B 161-163, 1143 (2000).

[27] J. Teichert, A. Büchner, P. Evtushenko, F. Gabriel, U. Lehnert, P. Michel, and J. Voigtländer, Nucl. Instrum. Methods Phys. Res. A 507, 354 (2003).

[28] R. Beyer, E. Birgersson, Z. Elekes, A. Ferrari, E. Grosse, R. Hannaske, A. R. Junghans, T. Kögler, R. Massarczyk, A. Matić, R. Nolte, R. Schwengner, and A. Wagner, Nucl. Instrum. Methods Phys. Res. A 723, 151 (2013).

[29] GSI Helmholtzzentrum für Schwerionenforschung $\mathrm{GmbH}$, Darmstadt, "Multi-Branch-System 5.0", https://www.gsi.de/ en/work/research/electronics/data_processing/data_acquisition/ mbs.htm (2006).

[30] X-5 Monte Carlo Team, MCNP - A General Monte Carlo N-Particle Transport Code, Version 5, Los Alamos National Laboratory (2003), 1A-UR-03-1987.

[31] W. Glöckle, H. Witała, D. Hüber, H. Kamada, and J. Golak, Phys. Rep. 274, 107 (1996). 